\title{
Analytic theory of curvature effects for wave problems with general boundary conditions
}

\author{
Willatzen, Morten; Gravesen, Jens; Voon, L. C. Lew Yan
}

Published in:

Physical Review A

Link to article, DOI:

10.1103/PhysRevA.81.060102

Publication date:

2010

Document Version

Publisher's PDF, also known as Version of record

Link back to DTU Orbit

Citation (APA):

Willatzen, M., Gravesen, J., \& Voon, L. C. L. Y. (2010). Analytic theory of curvature effects for wave problems with general boundary conditions. Physical Review A, 81(6), 060102.

https://doi.org/10.1103/PhysRevA.81.060102

\section{General rights}

Copyright and moral rights for the publications made accessible in the public portal are retained by the authors and/or other copyright owners and it is a condition of accessing publications that users recognise and abide by the legal requirements associated with these rights.

- Users may download and print one copy of any publication from the public portal for the purpose of private study or research.

- You may not further distribute the material or use it for any profit-making activity or commercial gain

- You may freely distribute the URL identifying the publication in the public portal

If you believe that this document breaches copyright please contact us providing details, and we will remove access to the work immediately and investigate your claim 


\title{
Analytic theory of curvature effects for wave problems with general boundary conditions
}

\author{
M. Willatzen* \\ Mads Clausen Institute for Product Innovation, University of Southern Denmark, Alsion 2, DK-6400 Sønderborg, Denmark \\ J. Gravesen \\ Department of Mathematics, Technical University of Denmark, DK-2800 Kongens Lyngby, Denmark
}

L. C. Lew Yan Voon

Department of Physics, Wright State University, 3640 Colonel Glenn Highway, Dayton, Ohio 45435, USA

(Received 17 November 2009; published 30 June 2010)

\begin{abstract}
A formalism based on a combination of differential geometry and perturbation theory is used to obtain analytic expressions for confined eigenmode changes due to general curvature effects. In cases of circular-shaped and helix-shaped structures, where alternative analytic solutions can be found, the perturbative solution is shown to yield the same result. The present technique allows the generalization of earlier results to arbitrary boundary conditions. The power of the method is illustrated using examples based on Maxwell's and Schrödinger's equations for applications in photonics and nanoelectronics.
\end{abstract}

DOI: 10.1103/PhysRevA.81.060102

The study of shape and curvature effects on physical properties is a problem that has similarities in many different fields of study in physics. Nanotechnology [1] has allowed a variety of complicated heterostructures to be grown, including helical and Möbius structures [2-7]. In photonics, electromagnetic modes have been confined in nanowires [8]. In plasmonics, electromagnetic propagation in bent nanowires is a current research area [9]. Nanopiezotronics involves electromechanical phenomena in bent nanowires [10]. In none of these cases has curvature effects been quantified. All the cases suggest developing a formalism for treating the phenomena either individually or coupled subject to distinct boundary conditions.

The methodology applied so far in the literature has been to identify the finite list of exactly solvable problems (for example, the 11 coordinate systems in which the Schrödinger equation is separable [11]) or in combination with differential geometry to identify quasiseparable problems in curved geometries [12]. Theoretical studies of shape effects for complicated structures, such as helical nanotubes [13], helicoidal ribbons and strips [14,15], elliptic tori [16], and a Möbius strip [17], have been published for the electronic problem subject to Dirichlet boundary conditions. In the present work, the method used in Ref. [12] is extended to encompass general boundary conditions and to provide analytic expressions for eigenmode changes due to general curvature effects based on a combination of differential geometry and perturbation theory. Perfect agreement is demonstrated versus alternative analytic results applicable in the case of circular- and helical-shaped nanotubes. The possibility of addressing Neumann boundary condition problems with the present method allows a different class of physics problems to be solved analytically, such as rigid-wall waveguide problems in acoustics and transverse electromagnetic wave propagation in hollow metallic waveguides.

*willatzen@mci.sdu.dk
PACS number(s): 03.65.Ge, 03.50.De

Consider a wire structure where the axis is given as a curve $\mathbf{x}(s)$ parametrized by arc length $s=u_{1}$. The tangent vector $\mathbf{t}(s)=\mathbf{x}^{\prime}(s)=d \mathbf{x} / d s$ is a unit vector field along the curve and we can augment it with vector fields $\mathbf{p}(s)$ and $\mathbf{q}(s)$ along the curve such that $\mathbf{t}(s), \mathbf{p}(s), \mathbf{q}(s)$ constitutes an orthonormal frame at each point $\mathbf{x}(s)$ along the axis.

Let $U \subseteq \mathbb{R}^{3}$ be a tubular neighborhood around the $x$ axis. Consider the corresponding tubular neighborhood around the curve $\mathbf{x}(s)$ parametrized by

$$
\tilde{\mathbf{x}}\left(s, u_{2}, u_{3}\right)=\mathbf{x}(s)+u_{2} \mathbf{p}(s)+u_{3} \mathbf{q}(s),\left(s, u_{2}, u_{3}\right) \in U,
$$

where $u_{2}$ and $u_{3}$ are small numbers. We then have

$$
\begin{gathered}
\mathbf{x}_{1}=\frac{\partial \tilde{\mathbf{x}}}{\partial s}=\mathbf{x}^{\prime}+u_{2} \mathbf{p}^{\prime}+u_{3} \mathbf{q}^{\prime} \\
=\left(1-a u_{2}-b u_{3}\right) \mathbf{t}-c u_{3} \mathbf{p}+c u_{2} \mathbf{q}, \\
\mathbf{x}_{2}=\frac{\partial \tilde{\mathbf{x}}}{\partial u_{2}}=\mathbf{p}, \\
\mathbf{x}_{3}=\frac{\partial \tilde{\mathbf{x}}}{\partial u_{3}}=\mathbf{q},
\end{gathered}
$$

where

$$
a(s)=\frac{d \mathbf{t}}{d s} \cdot \mathbf{p}, \quad b(s)=\frac{d \mathbf{t}}{d s} \cdot \mathbf{q}, \quad c(s)=\frac{d \mathbf{p}}{d s} \cdot \mathbf{q} .
$$

The metric tensor $\left(G_{i j}=\mathbf{x}_{i} \cdot \mathbf{x}_{j}\right)$ is

$$
\left[G_{i j}\right]=\left[\begin{array}{lrr}
\left(1-a u_{2}-b u_{3}\right)^{2} & -c u_{3} & c u_{2} \\
+\left(c u_{2}\right)^{2}+\left(c u_{3}\right)^{2} & & \\
-c u_{3} & 1 & 0 \\
c u_{2} & 0 & 1
\end{array}\right]
$$

The above formulation contains arbitrariness in the choice of $\mathbf{p}$ and $\mathbf{q}$ as a function of $u_{1}$. We use the so-called minimal rotation frame (MRF), where $\mathbf{p}$ and $\mathbf{q}$ are chosen such that $c=0$. In MRF, the Laplacian reads

$$
\begin{aligned}
\triangle & =G^{i j} \frac{\partial^{2}}{\partial u^{i} \partial u^{j}}+\left(G^{i j} \frac{\partial G}{2 G}+\frac{\partial G^{i j}}{\partial u^{j}}\right) \frac{\partial}{\partial u^{i}} \\
& =\left(\frac{\partial^{2}}{\partial\left(u_{2}\right)^{2}}+\frac{\partial^{2}}{\partial\left(u_{3}\right)^{2}}\right)-\left(a \frac{\partial}{\partial u_{2}}+b \frac{\partial}{\partial u_{3}}\right)
\end{aligned}
$$




$$
\begin{aligned}
& -\left(a^{2} u_{2} \frac{\partial}{\partial u_{2}}+b^{2} u_{3} \frac{\partial}{\partial u_{3}}+a b u_{2} \frac{\partial}{\partial u_{3}}+a b u_{3} \frac{\partial}{\partial u_{2}}\right) \\
& +\frac{\partial^{2}}{\partial s^{2}},
\end{aligned}
$$

where $G=\operatorname{det}\left(G_{i j}\right)$ and $G^{i j}=\left(G_{i j}\right)^{-1}$. Terms proportional to $\frac{\partial^{2}}{\partial\left(u_{2}\right)^{2}}, \frac{\partial}{\partial u_{2}}, u_{2} \frac{\partial}{\partial u_{2}}$ are kept, assuming small cross-sectional dimensions; similarly, for terms involving $u_{3}$ and derivatives in $u_{3}$ and mixed terms in $u_{2}, u_{3}$. We emphasize that these assumptions are the same as in earlier works [18-21], and that good agreement with exact results has been demonstrated in the Dirichlet case for a nanowire radius-to-curvature radius up to $10 \%$. [22]. A term proportional to $\left(u_{2}\right)^{2} \frac{\partial}{\partial u_{2}}$ is of order $u_{2}$ and hence negligible for curved structures with large aspect ratios (small cross-sectional dimensions compared to the length dimension).

We first present the theory for transverse-electric (TE) electromagnetic propagation in a curved metallic-covered nanostructure where Neumann boundary conditions apply. The wave equation is

$$
\left(\nabla^{2}+\mu \epsilon \omega^{2}\right) H_{3}=0,
$$

where $H_{i}$ is the Hertz vector component along $u_{i}(i=1,2,3)$, $\frac{\omega}{2 \pi}$ is the wave frequency, $\mu$ is the permeability, and $\epsilon$ is the permittivity. The TE boundary condition for a plane curved nanowire center-line curve is

$$
\frac{\partial H_{3}}{\partial n}=0,
$$

where $n$ denotes the boundary normal.

In the particular case of a center-line curve located in the $u_{1}-u_{2}$ plane, the Laplacian curvature contribution $W$ reads in $\operatorname{MRF}\left[c=0, a=\kappa\left(u_{1}\right), b=0\right]$ [23]

$$
W=-\kappa\left(u_{1}\right) \frac{\partial}{\partial u_{2}}-\kappa\left(u_{1}\right)^{2} u_{2} \frac{\partial}{\partial u_{2}},
$$

where $\kappa\left(u_{1}\right)$ is the centerline curvature. We then have

$$
\begin{aligned}
\Delta\left(-\mu \epsilon \omega_{n l m}^{2}\right) & =\langle n l m|W| n l m\rangle+\sum_{n^{\prime} l^{\prime} m^{\prime}} \frac{\left|\left\langle n l m|W| n^{\prime} l^{\prime} m^{\prime}\right\rangle\right|^{2}}{\mu \epsilon \omega_{n l m}^{2}-\mu \epsilon \omega_{n l m, 0}^{2}} \\
& =-\frac{3}{4}\left(1-\delta_{l 0}\right) \kappa^{2},
\end{aligned}
$$

where the latter equality applies to the constant curvature case keeping terms up to second order in the curvature [23], $\delta_{l 0}=1$ if $l=0\left(\delta_{l 0}=0\right.$ if $\left.l \neq 0\right)$, and $|n l m\rangle$ is an unperturbed eigenstate. In Eq. (11), $n, l, m$ are integers corresponding to the unperturbed separable wave parts in $u_{1}, u_{2}, u_{3}$, respectively. For example, the unperturbed $u_{2}$ wave parts in the case with Neumann boundary conditions are

$$
\begin{aligned}
& |2 l\rangle=\frac{1}{\sqrt{\left(1+\delta_{l 0}\right) \varepsilon_{2}}} \cos \left(\frac{2 l \pi}{2 \varepsilon_{2}} u_{2}\right), \\
& |2 l+1\rangle=\frac{1}{\sqrt{\varepsilon_{2}}} \sin \left(\frac{(2 l+1) \pi}{2 \varepsilon_{2}} u_{2}\right),
\end{aligned}
$$

where $l=0,1,2, \ldots$, and $2 \varepsilon_{2}$ defines the nanowire $u_{2}$ range: $-\varepsilon_{2} \leqslant u_{2} \leqslant \varepsilon_{2}$. As a corollary, for plane center-line curves, Eq. (11) shows that there is no eigenvalue shift for states with $l=0$ in the Neumann boundary case as the corresponding unperturbed solution is independent of $u_{2}$ [Eq. (12)]. The result now follows as matrix elements of the perturbation $W$ between $l=0$ states vanish identically according to Eq. (10). For all other states $(l \neq 0)$, there is a shift in frequency given by $\Delta \omega_{n l m}^{2}=\frac{3}{4} \frac{\kappa^{2}}{\mu \epsilon}$. Evidently, the strength of the present perturbative differential-geometry procedure is that it can be easily carried out in the general case with a nonplanar center-line curve subject to a varying curvature.

Combining Eq. (11) with the unperturbed eigenfrequencies $\omega_{0, n l m}$, we obtain

$$
\frac{\Delta \omega_{n l m}^{2}}{\omega_{0, n l m}^{2}}=\frac{3 \kappa^{2}}{4} \frac{\left(1-\delta_{l 0}\right)}{\left(\frac{n \pi}{L}\right)^{2}+\left(\frac{l \pi}{2 \varepsilon_{2}}\right)^{2}+\left(\frac{m \pi}{2 \varepsilon_{3}}\right)^{2}},
$$

where $-\varepsilon_{3} \leqslant u_{3} \leqslant \varepsilon_{3}$ defines the nanowire $u_{3}$ range. We now apply the theory to a metallic-dielectric coaxial structure, where the dielectric is a Si nanowire similar to those considered experimentally by Tong et al. [24] with $2 \varepsilon_{2}=800 \mathrm{~nm}$ and a sharp circular-shaped bend $(R=800 \mathrm{~nm})$. We find a relative eigenfrequency shift for the mode with $l=1, m=0, n=0$ equal to

$$
\frac{\Delta \omega_{010}^{2}}{\omega_{0,010}^{2}}=\frac{3}{\pi^{2}}\left(\frac{\varepsilon_{2}}{R}\right)^{2}=0.076
$$

(i.e., a non-negligible $8 \%$ ). This example provides a clear picture of the importance of curvature for this type of structures.

As a second example, we consider a nanowire one-particle Schrödinger equation. A general parametrization gives

$$
\begin{gathered}
H \psi=E \psi, H=H_{0}+W \\
H_{0}=-\frac{\hbar^{2}}{2 m_{0}}\left(\frac{\partial^{2}}{\partial\left(u_{2}\right)^{2}}+\frac{\partial^{2}}{\partial\left(u_{3}\right)^{2}}+\frac{\partial^{2}}{\partial t^{2}}\right)+V(\mathbf{r}) \\
W=-\frac{\hbar^{2}}{2 m_{0}}\left[-\left(a \frac{\partial}{\partial u_{2}}+b \frac{\partial}{\partial u_{3}}\right)\right. \\
-\left(a^{2} u_{2} \frac{\partial}{\partial u_{2}}+b^{2} u_{3} \frac{\partial}{\partial u_{3}}+a b u_{2} \frac{\partial}{\partial u_{3}}+a b u_{3} \frac{\partial}{\partial u_{2}}\right) \\
\left.+\left(\frac{1}{\mathbf{x}^{\prime} \cdot \mathbf{x}^{\prime}}-1\right) \frac{\partial^{2}}{\partial t^{2}}-\frac{\mathbf{x}^{\prime} \cdot \mathbf{x}^{\prime \prime}}{\left(\mathbf{x}^{\prime} \cdot \mathbf{x}^{\prime}\right)^{2}} \frac{\partial}{\partial t}\right]
\end{gathered}
$$

where $t$ is the coordinate for the parametrization of the nanowire center line, $\hbar$ is Planck's constant divided by $2 \pi, m_{0}$ is the particle mass, and $V(\mathbf{r})$ is the potential (assumed to be zero inside the nanowire). The renormalized wave function used in, e.g., Refs. [19,20], differs from our unrenormalized wave function $\psi$ by a factor of $\sqrt{G}$. We emphasize the advantage in performing a perturbative analysis in $\psi$ since a Neumann boundary condition problem in $\psi$ does not correspond to a simple Neumann problem in the renormalized wave function used in Refs. [19,20].

Since eigenstates for the unperturbed problem are parity eigenstates, terms changing parity in one or more coordinates, such as $a \frac{\partial}{\partial u_{2}}$, do not contribute in first-order perturbation theory but they do contribute in second order. Eigenvalue contributions to lowest order in $a$ (or $b$ ) cannot occur and contributions to higher order than $a^{2}$, etc. are too small and will be discarded henceforth. Thus, the additional terms in the 
Laplacian involving $a$ or $b$ lead to energy eigenvalue changes $\Delta E_{n l m}$ in the unperturbed eigenstate $\left.|n| m\right\rangle$ given by

$$
\begin{aligned}
\Delta E_{n l m}= & \frac{\hbar^{2}}{2 m_{0}}\left\langle n l m\left|\left(a^{2} u_{2} \frac{\partial}{\partial u_{2}}+b^{2} u_{3} \frac{\partial}{\partial u_{3}}\right)\right| n l m\right\rangle \\
& +\frac{\hbar^{4}}{4 m_{0}^{2}} \sum_{n^{\prime} l^{\prime} m^{\prime}}^{\prime} \frac{\left|\left\langle n l m\left|\left(a \frac{\partial}{\partial u_{2}}+b \frac{\partial}{\partial u_{3}}\right)\right| n^{\prime} l^{\prime} m^{\prime}\right\rangle\right|^{2}}{E_{n^{\prime} l^{\prime} m^{\prime}}^{0}-E_{n l m}^{0}} \\
& -\frac{\hbar^{2}}{2 m_{0}}\left\langle n l m\left|\left(\frac{1}{\mathbf{x}^{\prime} \cdot \mathbf{x}^{\prime}}-1\right) \frac{\partial^{2}}{\partial t^{2}}-\frac{\mathbf{x}^{\prime} \cdot \mathbf{x}^{\prime \prime}}{\left(\mathbf{x}^{\prime} \cdot \mathbf{x}^{\prime}\right)^{2}} \frac{\partial}{\partial t}\right| n l m\right\rangle \\
& -\frac{\hbar^{4}}{4 m_{0}^{2}} \sum_{n^{\prime} l^{\prime} m^{\prime}}^{\prime} \frac{\left|\left\langle n l m\left|\left(\frac{1}{\mathbf{x}^{\prime} \cdot \mathbf{x}^{\prime}}-1\right) \frac{\partial^{2}}{\partial t^{2}}-\frac{\mathbf{x}^{\prime} \cdot \mathbf{x}^{\prime \prime}}{\left(\mathbf{x}^{\prime} \cdot \mathbf{x}^{\prime}\right)^{2}} \frac{\partial}{\partial t}\right| n^{\prime} l^{\prime} m^{\prime}\right\rangle\right|^{2}}{E_{n^{\prime} l^{\prime} m^{\prime}}^{0}-E_{n l m}^{0}},
\end{aligned}
$$

where $E_{n l m}^{0}$ is the unperturbed eigenvalue, and $n, l, m$ are the quantum indices associated with the $u_{1}, u_{2}, u_{3}$ coordinates, respectively. Hence, it is possible to obtain the influence of bending effects on eigenvalues of a general bent structure by solving (computationally fast) integrals in known unperturbed eigenstates. From now on, only structures with rectangular cross sections will be considered for concreteness. A normalized unperturbed Dirichlet eigenstate $|n l m\rangle$ is

$$
|n l m\rangle \equiv \psi_{n l m}=\psi_{n}^{1} \psi_{l}^{2} \psi_{m}^{3}
$$

where

$$
\begin{gathered}
\psi_{n}^{1}\left(u_{1}\right)=\sqrt{\frac{2}{L}} \sin \left(\frac{n \pi}{L} u_{1}\right), \\
\psi_{2 l-1}^{i}=\frac{1}{\sqrt{\varepsilon_{i}}} \cos \left(\frac{(2 l-1) \pi}{2 \varepsilon_{i}} u_{i}\right), \\
\psi_{2 l}^{i}=\frac{1}{\sqrt{\varepsilon_{i}}} \sin \left(\frac{l \pi}{\varepsilon_{i}} u_{i}\right),
\end{gathered}
$$

with $i=2,3 ; n, l, m=1,2, \ldots ; L$ defines the $u_{1}$ range $0 \leqslant$ $u_{1} \leqslant L$, and $2 \varepsilon_{i}$ defines the range of $u_{i}:-\varepsilon_{i} \leqslant u_{i} \leqslant \varepsilon_{i}$.

The first-order perturbation contribution proportional to $a^{2}$ is [from Eq. (18)]

$$
\Delta E_{n l m}^{(1 a)}=\frac{1}{2}\left(-\frac{\hbar^{2}}{2 m_{0}}\right) \int_{0}^{L} \psi_{n}^{1}\left(u_{1}\right) a\left(u_{1}\right)^{2} \psi_{n}^{1}\left(u_{1}\right) d u_{1} .
$$

The other terms in Eq. (18) are similarly obtained.

In the case of an arc-length parametrized circular-bent or helical-bent structure with constant curvature $\kappa$, an analytic evaluation of the four perturbative contributions in Eq. (18) gives the following result, independent of the quantum indices to second order in the curvature:

$$
\Delta E_{n l m}=\left(-\frac{\hbar^{2}}{2 m_{0}}\right) \frac{\kappa^{2}}{4} .
$$

The present result, obtained perturbatively, agrees with the result obtained in previous works [12,18-20,22]. We emphasize that Eq. (18) applies to cases with general boundary conditions (e.g., Dirichlet, Neumann, or Robin) while previous works [12,17-20,22] require Dirichlet conditions. For a general bent structure, a simple analytic result as Eq. (24) cannot be found but the fast computation of eigenstate and

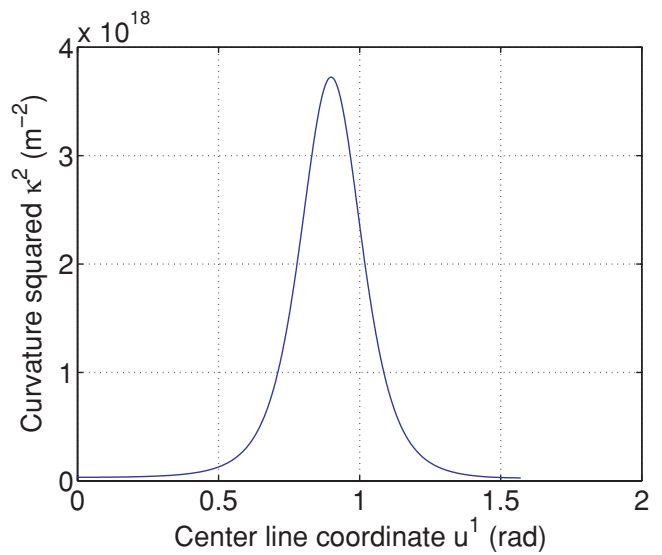

FIG. 1. (Color online) Squared curvature for a Möbius-shaped nanowire structure with parameters $c_{1}=3 \mathrm{~nm}, c_{2}=2 \mathrm{~nm}$, and $c_{3}=$ $0.5 \mathrm{~nm}$.

eigenvalue changes for bent structures based on Eq. (18) still applies.

The above result applies to the $\mathrm{ZnO}$ ring structure examined experimentally by Kong et al. [25]. For $R=\frac{1}{\kappa}=0.5 \mu \mathrm{m}$, $2 \varepsilon_{2}=30 \mathrm{~nm}, m_{0}=0.27$ (in units of the free-electron mass),
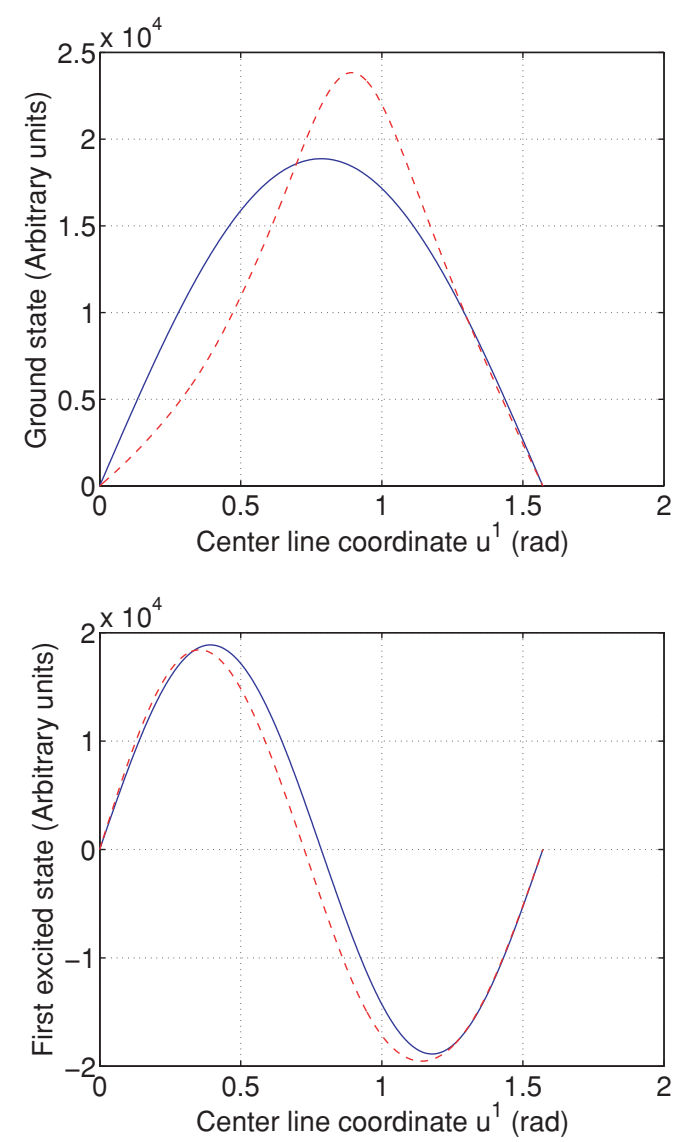

FIG. 2. (Color online) Ground-state (top) and first-excited (bottom) states of a Möbius-shaped nanowire with parameters $c_{1}=3$ $\mathrm{nm}, c_{2}=2 \mathrm{~nm}$, and $c_{3}=0.5 \mathrm{~nm}$. Solid and dashed lines indicate the unperturbed and perturbed eigenstates, respectively. 
TABLE I. Energy shifts due to bending for a Möbius-shaped nanowire. Values are in $\mathrm{meV}$.

\begin{tabular}{lcc}
\hline \hline$\Delta E_{1}$ (ground) & $\Delta E_{2}$ (first) & $\Delta E_{3}$ (second) \\
\hline-121.5 & -62.45 & -299.1 \\
\hline \hline
\end{tabular}

and $2 \varepsilon_{3}=1 \mu \mathrm{m}$, we find

$$
\Delta E_{011}=-1.4 \times 10^{-4} \mathrm{meV}, \quad E_{011}=1.55 \mathrm{meV} .
$$

In other words, the shift in energy is negligible for these rather big nanostructures. The theory also allows an analytic calculation of the energy shift for nanohelices [25], a result that could otherwise only be obtained numerically.

Finally, as an illustration of a nontrivial problem, the formalism is applied to a Möbius-shaped nanowire structure. The parametrization is given by

$$
\mathbf{x}\left(u_{1}\right)=\left(c_{1} \sin \left(u_{1}\right), \quad c_{2} \sin \left(2 u_{1}\right), \quad c_{3} \cos \left(3 u_{1}\right)\right),
$$

where $c_{i}$ are coefficients. We choose $c_{1}=3 \mathrm{~nm}, c_{2}=2 \mathrm{~nm}$, $c_{3}=0.5 \mathrm{~nm}$, and assume small cross-sectional dimensions compared to the center-line length. In Fig. 1, the squared curvature is plotted versus $u_{1}$ corresponding to a nanowire length equal to one-quarter of a full Möbius period (i.e., for a parameter interval, $0 \leqslant u_{1} \leqslant \pi / 2$ ). The $u_{1}$ dependence of the ground and first excited states is shown in Fig. 2. We emphasize that the assumption of small cross-sectional dimensions ensures that significant perturbative wave-function corrections have the same $u_{2}$ and $u_{3}$ functional dependence as the corresponding unperturbed wave function. Wave-function perturbations are significant mainly where the curvature is highest as expected. The corresponding changes in meV are shown in Table I. For the ground state, the first three contributions in Eq. (18) to the change in energy are -0.358 , 0.179 , and $0.058 \mathrm{eV}$, respectively, with the fourth contribution being negligible. The large curvature is seen to have a significant impact on the eigenvalues. Furthermore, one can explain why the energy change for the first excited state is relatively smaller, as the wave function has a zero near the region of high curvature. Our study reveals that curvature can be an important tool in controlling the electronic properties of nanostructures.

In summary, an analytic method for evaluating the influence of curvature effects on three-dimensional eigenmodes is presented. The method is able to treat arbitrary boundary conditions by using a methodology of combining differential geometry with perturbation theory. Results are in perfect agreement with alternative analytic methods applicable only in the cases of simple curved structures. The technique presented here allows one to quickly and accurately quantify the effect of curvature for a variety of physical phenomena and shapes. The formalism is applicable to structures with large aspect ratios but generalizable to cases where this assumption is relaxed. Thus, it was shown that curvature effects have little influence on the electronic properties of current $\mathrm{ZnO}$ nanorings and nanohelices [25] but are not negligible for the electromagnetic properties of curved Si waveguides [24]. Even more pronounced curvature effects were predicted for Möbius-type structures [4]. The present work opens up a whole new field of analysis of coupled multiphysics phenomena of complex three-dimensional structures subject to arbitrary boundary conditions.
[1] T. Ando, A. Fowler, and F. Stern, Rev. Mod. Phys. 54, 437 (1992).

[2] V. Ya Prinz, D. Grutzmacher, A. Beyer, C. David, and B. Ketterer, Nanotechnol. 12, S1 (2001).

[3] O. G. Schmidt and K. Eberl, Nature (London) 410, 168 (2001).

[4] S. Tanda, T. Tsuneta, Y. Okajima, K. Inagaki, K. Yamaya, and N. Hatakenaka, Nature (London) 417, 397 (2002).

[5] Z. Hens, D. Vanmaekelbergh, E. J. A. J. Stoffels, and H. van Kempen, Phys. Rev. Lett. 88, 236803 (2002).

[6] X. Duan, C. Niu, V. Sahi, J. Chen, J. W. Parce, S. Empedocles, and J. L. Goldman, Nature (London) 425, 274 (2003).

[7] P. X. Gao, Y. Ding, W. Mai, W. L. Hughes, C. Lao, and Z. L. Wang, Science 309, 1700 (2005).

[8] M. H. Huang, S. Mao, H. Feick, H. Yan, Y. Wu, H. Kind, E. Weber, R. Russo, and P. Yang, Science 292, 1897 (2001).

[9] S. Lal, S. Link, and N. J. Halas, Nat. Phot. 1, 641 (2007).

[10] Z. L. Wang, Adv. Mater. 19, 889 (2007).

[11] L. P. Eisenhart, Ann. Math. 35, 284 (1934).
[12] J. Gravesen and M. Willatzen, Physica B 371, 112 (2006).

[13] V. Atanasov and R. Dandoloff, Phys. Lett. A 372, 6141 (2008).

[14] R. Dandoloff and T. T. Truong, Phys. Lett. A 325, 233 (2004).

[15] E. L. Starostin and G. H. M. van der Heijden, Phys. Rev. Lett. 101, 084301 (2008).

[16] M. Encinosa and M. Jack, Phys. Scr. 73, 439 (2006).

[17] J. Gravesen and M. Willatzen, Phys. Rev. A 72, 032108 (2005).

[18] H. Jensen and H. Koppe, Ann. Phys. (Leipzig) 63, 586 (1977).

[19] R. C. T. da Costa, Phys. Rev. A 23, 1982 (1981).

[20] R. C. T. da Costa, Phys. Rev. A 25, 2893 (1982).

[21] J. Goldstone and R. L. Jaffe, Phys. Rev. B 45, 14100 (1992).

[22] J. Gravesen, M. Willatzen, and L. C. Lew Yan Voon, J. Math. Phys. 46, 012107 (2005).

[23] M. Willatzen, J. Gravesen, and L. C. Lew Yan Voon (unpublished).

[24] L. Tong, J. Lou, R. R. Gattass, S. He, X. Chen, L. Liu, and E. Mazur, Nano Lett. 5, 259 (2005).

[25] X. Y. Kong, Y. Ding, R. Yang, and Z. L. Wang, Science 303, 1348 (2004). 